TRANSACTIONS OF THE

AMERICAN MATHEMATICAL SOCIETY

Volume 360, Number 5, May 2008, Pages 2681-2692

S 0002-9947(07)04437-6

Article electronically published on December 20, 2007

\title{
HIGHER ORDER PDE'S AND ITERATED PROCESSES
}

\author{
ERKAN NANE
}

\begin{abstract}
We introduce a class of stochastic processes based on symmetric $\alpha$-stable processes, for $\alpha \in(0,2]$. These are obtained by taking Markov processes and replacing the time parameter with the modulus of a symmetric $\alpha$-stable process. We call them $\alpha$-time processes. They generalize Brownian time processes studied in Allouba and Zheng (2001), Allouba (2002), (2003), and they introduce new interesting examples. We establish the connection of $\alpha$-time processes to some higher order PDE's for $\alpha$ rational. We also obtain the PDE connection of subordinate killed Brownian motion in bounded domains of regular boundary.
\end{abstract}

\section{INTRODUCTION}

The link between concepts from probability and partial differential equations (PDE's) helped solve problems in analysis or find easier and shorter proofs for well-known results. Researchers have been fascinated by these kinds of links. The classical well-known connection of a PDE and a stochastic process is the Brownian motion and heat equation connection. Let $X_{t} \in \mathbb{R}^{n}$ be the Brownian motion started at $x$. Then the function

$$
u(t, x)=E_{x}\left[f\left(X_{t}\right)\right]
$$

solves the Cauchy problem

$$
\begin{aligned}
\frac{\partial}{\partial t} u(t, x) & =\Delta u(t, x), \quad t>0, \quad x \in \mathbb{R}^{n}, \\
u(0, x) & =f(x), \quad x \in \mathbb{R}^{n} .
\end{aligned}
$$

In recent years, starting with the articles of Burdzy $[4,5]$, researchers had interest in iterated processes in which one changes the time parameter with one-dimensional Brownian motion; see $[1,3,4,5,6,9,10,12,16]$ and references there in. The connections of iterated Brownian motion (IBM) $Z_{t}=X\left(Y_{t}\right)$, where $X$ is a two-sided Brownian motion and $Y$ is another Brownian motion independent of $X$, and the biLaplacian have been found by Allouba and Zheng [1] in (2001) and by DeBlassie [9] in (2004). They showed that the function

$$
u(t, x)=E_{x}\left[f\left(Z_{t}\right)\right]
$$

Received by the editors May 8, 2006.

2000 Mathematics Subject Classification. Primary 60J65, 60K99.

Key words and phrases. Iterated Brownian motion, exit time, PDE connection, $\alpha$-stable process, $\alpha$-time process, subordinate killed Brownian motion.

This work was supported in part by NSF Grant \# 9700585-DMS.. 
solves the Cauchy problem

$$
\begin{aligned}
\frac{\partial}{\partial t} u(t, x) & =\frac{\Delta f(x)}{\sqrt{\pi t}}+\Delta^{2} u(t, x), \quad t>0, \quad x \in \mathbb{R}^{n}, \\
u(0, x) & =f(x), \quad x \in \mathbb{R}^{n} .
\end{aligned}
$$

The non-Markovian property of IBM is reflected by the appearance of the initial function $f(x)$ in the PDE.

The results in the above mentioned articles are a motivation for us to define and study our processes in this paper. Our aim in this paper is twofold. In the first part we define $\alpha$-time processes and study their PDE connection. In the second part, we study the PDE connection of subordinate killed Brownian motion over bounded domains of regular boundary.

In analogy with the Brownian time processes (BTP)'s studied in [1, 2], we define the ' $\alpha$-time' processes and establish the connection of these processes to several higher order PDE's. Since the time clock that will replace ordinary time is a symmetric $\alpha$-stable process, we first give the general definition for these processes.

The $n$-dimensional symmetric stable process $X_{t}$ with index $\alpha \in(0,2]$ is the process with stationary independent increments whose transition density

$$
p_{t}^{\alpha}(x, y)=p^{\alpha}(t, x-y), \quad(t, x, y) \in(0, \infty) \times \mathbb{R}^{n} \times \mathbb{R}^{n},
$$

is characterized by the Fourier transform

$$
\int_{\mathbb{R}^{n}} e^{i y \cdot \xi} p^{\alpha}(t, y) d y=\exp \left(-t|\xi|^{\alpha}\right), \quad t>0, \xi \in \mathbb{R}^{n} .
$$

The process has right continuous paths, and it is rotation and translation invariant. For $\alpha=2$, this is Brownian motion.

We now give the definitions of $\alpha$-time processes and some other iterated processes. Let $\alpha \in(0,2]$. Let $Y(t)$ be a 1 -dimensional $\alpha$-stable process independent of the continuous Markov process $X^{x}(t)$, started at 0 , and define the ' $\alpha$-time' process $\alpha \mathrm{TP}$ by $Z_{Y}^{x}(t):=X^{x}(|Y(t)|)$. Similarly, we define $\mathrm{kE} \alpha \mathrm{TP}$ the excursions based ' $\alpha$-time' process $Z_{Y, c}^{x, k}$; from $\alpha$ TP's by breaking up the path of $|Y(t)|$ into excursion intervals-maximal intervals $(r, s)$ of time on which $|Y(t)|>0$-and, on each such interval, we pick an independent copy of the Markov process $X^{x}$ from a finite or an infinite collection. Let $X^{x, 1}(t) \cdots X^{x, k}(t)$ be independent copies of $X^{x}(t)$ starting from the point $x$. On each excursion interval of $|Y(t)|$ use one of the $k$ copies chosen at random. For $\alpha=2$, these are the Brownian time processes defined in $[1,2,3]$. When the outer Markov process is a Brownian motion, $\alpha=2$ and $k=2$, this is the famous iterated Brownian motion of Burdzy [4]. We will study PDE connection of these processes extensively in sections 2-3.

In section 4, we will study subordinate killed Brownian motion, which can be realized as a composition of Brownian motion killed on the boundary of regular domain and a subordinator. Let $D \subset \mathbb{R}^{n}$ be a bounded domain and let $X_{t}^{D}$ be the Brownian motion killed upon exiting $D$. The subordinate killed Brownian motion $Z_{t}^{D}$ is defined as the process obtained by subordinating $X^{D}$ via the $\alpha / 2$ subordinator $T_{t}$. More precisely, let $Z_{\alpha}^{D}=X^{D}\left(T_{t}\right), t>0$. Then $Z_{\alpha}^{D}$ is a Hunt process on $D$. If we use $\left(P_{t}^{D}\right)_{t \geq 0}$ to denote the semigroup of $X^{D}$, then the semigroup $Q_{t}^{\alpha}$ of $Z_{\alpha}^{D}$ is given by

$$
Q_{t}^{\alpha} f(x)=\int_{0}^{\infty} P_{s}^{D} f(x) u_{t}^{\alpha / 2}(s) d s
$$


Potential theory of this process has been studied by R. Song and Z. Vodraček in [15] over Lipschitz domains and $C^{1,1}$ domains. In particular, they established intrinsic ultraconractivity of the semigroup of this process and bounds on the Green function and the jumping kernel.

R. Song in [14] established sharp bounds on the density, Green function and the jumping function of subordinate killed Brownian motion. For other properties of subordinate killed Brownian motion check the references in [14] and [15].

Our proofs follow the main ideas of Allouba and Zheng [1], Allouba [2, 3] and DeBlassie [8], with some crucial changes (see Lemma 3.1 and Lemma 3.3). Our main contribution is seeing that Markov processes can be subordinated by symmetric stable processes to give new interesting types of processes. The new technique in our proofs is using higher order PDE's satisfied by transition density of symmetric $\alpha$-stable processes for $\alpha$ rational, Lemma 3.2: for example transition density of a real-valued Cauchy process satisfies wave equation.

The paper is organized as follows. We give the main results and proofs of PDE connection of $\alpha$-time processes in $\S 2$. We collect some useful lemmas in $\S 3$. In $\S 4$, we study the PDE connection of subordinate killed Brownian motion in bounded domains of regular boundary.

\section{2. $\alpha$-TIME PROCESSES}

The first PDE connection of ' $\alpha$-time' processes is the following. When $\alpha=1$, we call this process a Cauchy-time process $(\mathrm{CTP})$ and denote it by $Z_{C}^{x}(t)$, for $C(t)$ a real-valued Cauchy process.

Theorem 2.1. Let $\mathcal{T}_{s} f(x)=E\left[f\left(X^{x}(s)\right)\right]$ be the semigroup of the continuous Markov process $X^{x}(t)$ and let $\mathcal{A}$ be its generator. Let $\alpha=1$. Let $f$ be a bounded measurable function in the domain of $\mathcal{A}$, with $D_{i j} f$ bounded and Hölder continuous for all $1 \leq i, j \leq n$. If $u(t, x)=E\left[f\left(Z_{C, c}^{x, k}(t)\right)\right]$ for any $k \in \mathbb{N}$, then u solves

$$
\begin{aligned}
\frac{\partial^{2}}{\partial t^{2}} u(t, x) & =-\frac{2 \mathcal{A} f(x)}{\pi t}-\mathcal{A}^{2} u(t, x), \quad t>0, \quad x \in \mathbb{R}^{n}, \\
u(0, x) & =f(x), \quad x \in \mathbb{R}^{n},
\end{aligned}
$$

where the operator $\mathcal{A}$ acts on $u(t, x)$ as a function of $x$ with $t$ fixed. In particular, if $X^{x}(t)$ is Brownian motion started at $x$ and $\Delta$ is the standard Laplacian, then $u$ solves

$$
\begin{aligned}
\frac{\partial^{2}}{\partial t^{2}} u(t, x) & =-\frac{2 \Delta f(x)}{\pi t}-\Delta^{2} u(t, x), \quad t>0, \quad x \in \mathbb{R}^{n}, \\
u(0, x) & =f(x), \quad x \in \mathbb{R}^{n} .
\end{aligned}
$$

Remark 2.1. Notice the effect of the initial function is different than the Brownian motion heat equation case; it is very similar to the effect of the initial function in IBM-PDE connection. The appearance of the initial function in the PDE reflects the non-Markovian property of the iterated processes. This PDE is very similar to the one in [1, Theorem 0.1] in that this has the same order space derivatives (i.e. Laplacian and biLaplacian), but on the other hand this has second order time derivative and the coefficients are different.

Proof. We first consider the process $Z_{C}^{x}$. We use the representation

$$
u(t, x)=E\left[f\left(Z_{C}^{x}(t)\right)\right]=2 \int_{0}^{\infty} p_{t}^{1}(0, s) \mathcal{T}_{s} f(x) d s,
$$


where $p_{t}^{1}(0, s)=\frac{t}{\pi\left(s^{2}+t^{2}\right)}$ is the transition density of the Cauchy process on $\mathbb{R}$. Using dominated convergence and the fact that $p_{t}^{1}(0, s)$ satisfy

$$
\left(\frac{\partial^{2}}{\partial s^{2}}+\frac{\partial^{2}}{\partial t^{2}}\right) p_{t}^{1}(0, s)=0
$$

we obtain

$$
\begin{aligned}
\frac{\partial^{2}}{\partial t^{2}} E\left[f\left(Z_{C}^{x}(t)\right)\right] & =2 \int_{0}^{\infty} \frac{\partial^{2}}{\partial t^{2}} p_{t}^{1}(0, s) \mathcal{T}_{s} f(x) d s \\
& =2 \int_{0}^{\infty}-\frac{\partial^{2}}{\partial s^{2}} p_{t}^{1}(0, s) \mathcal{T}_{s} f(x) d s
\end{aligned}
$$

We now integrate by parts twice and observe that boundary terms always vanish at $\infty($ as $s \rightarrow \infty)$ and we have $\frac{\partial}{\partial s} p_{t}^{1}(0,0)=0$, but $p_{t}^{1}(0,0)>0$. Thus

$$
\begin{aligned}
\frac{\partial^{2}}{\partial t^{2}} E\left[f\left(Z_{C}^{x}(t)\right)\right] & =2 \int_{0}^{\infty} \frac{\partial}{\partial s} p_{t}^{1}(0, s) \frac{\partial}{\partial s} \mathcal{T}_{s} f(x) d s \\
& =-2 p_{t}^{1}(0,0) \mathcal{A} f(x)-2 \int_{0}^{\infty} p_{t}^{1}(0, s) \mathcal{A}^{2} \mathcal{T}_{s} f(x) d s .
\end{aligned}
$$

Taking the application of $\mathcal{A}^{2}$ outside the integral using the conditions on $f$ and $D_{i j} f$ by Lemma 3.1 we get

$$
\frac{\partial^{2}}{\partial t^{2}} u(t, x)=-2 p_{t}^{1}(0,0) \mathcal{A} f(x)-\mathcal{A}^{2} u(t, x) .
$$

For the other processes, by similar arguments as in Theorem 0.1 in Allouba and Zheng [1], considering the excursion intervals of $|C(t)|$ we have

$$
E\left[f\left(Z_{C}^{x}(t)\right)\right]=E\left[f\left(Z_{C, c}^{x, k}(t)\right)\right] .
$$

We provide the argument in Allouba and Zheng for completeness. Let $e^{-}(t)$ be the $|C(t)|$-excursion immediately preceding the excursion straddling $t, e(t)$, on the condition that we pick the $j$ th copy of $X^{x}$ on $e^{-}(t)$ (uniformly among the $k$ copies of $\left.X^{x}\right)$, using the independence of the process $X^{x, j}$ on $e^{-}(t)$ from $C(t)$ and from the following choice of $X^{x}$ copy on $e(t)$ to get

$$
\begin{aligned}
E\left[f\left(Z_{C, c}^{x, k}(t)\right)\right] & =2 \sum_{j=1}^{k} \int_{0}^{\infty} p_{t}^{1}(0, s) \mathcal{T}_{s} f(x) P\left[\text { picking the } j \text { th copy on } e^{-}(t)\right] d s \\
& =\frac{2}{k} \sum_{j=1}^{k} \int_{0}^{\infty} p_{t}^{1}(0, s) \mathcal{T}_{s} f(x) d s=2 \int_{0}^{\infty} p_{t}^{1}(0, s) \mathcal{T}_{s} f(x) d s \\
& =E\left[f\left(Z_{C}^{x}(t)\right)\right] .
\end{aligned}
$$

This concludes the proof of Theorem 2.1.

Next, we solve a similar PDE as in [2, Theorem 1.2] which is obtained by running an $\epsilon$-scaled CTP and averaging the product of $f\left(Z_{\epsilon C, c}^{x, k}(t)\right)$ with the negative exponential of $|C(t)| / \epsilon$, for $C(t)$ a real-valued Cauchy process. This looks like the Feynman-Kac formula when $\epsilon=1$. We state this theorem since it is a step towards the probabilistic study of Linearized Cahn-Hilliard and Kuramoto-Sivashinsky type PDE's as in [2]. 
Theorem 2.2. Under the same conditions on $f$ as in Theorem 2.1, and for $\epsilon>0$, if

$$
u_{\epsilon}(t, x)=E\left[f\left(Z_{\epsilon C, c}^{x, k}(t)\right) \exp \left(-\frac{|C(t)|}{\epsilon}\right)\right]
$$

for any $k \in \mathbb{N}$, then $u_{\epsilon}$ solves

$$
\left\{\begin{aligned}
\frac{\partial^{2}}{\partial t^{2}} u_{\epsilon}(t, x) & =-\frac{2}{\pi t}\left[\epsilon \mathcal{A} f(x)-\frac{1}{\epsilon} f(x)\right]-\frac{1}{\epsilon^{2}} u_{\epsilon}(t, x) & & \\
& +2 \mathcal{A} u_{\epsilon}(t, x)-\epsilon^{2} \mathcal{A}^{2} u_{\epsilon}(t, x), & & t>0, x \in \mathbb{R}^{n}, \\
u_{\epsilon}(0, x) & =f(x)=\lim _{t \downarrow 0, y \rightarrow x} u_{\epsilon}(t, y), & & x \in \mathbb{R}^{n} .
\end{aligned}\right.
$$

In particular, If the outer process $X^{x}$ in $(2.5)$ is a Brownian motion, then $u_{\epsilon}(t, x)$ solves

$$
\left\{\begin{aligned}
\frac{\partial^{2}}{\partial t^{2}} u_{\epsilon}(t, x) & =-\frac{2}{\pi t}\left[\epsilon \Delta f(x)-\frac{1}{\epsilon} f(x)\right]-\frac{1}{\epsilon^{2}} u_{\epsilon}(t, x) & & \\
& +2 \Delta u_{\epsilon}(t, x)-\epsilon^{2} \Delta^{2} u_{\epsilon}(t, x), & & t>0, x \in \mathbb{R}^{n}, \\
u_{\epsilon}(0, x) & =f(x)=\lim _{t \downarrow 0, y \rightarrow x} u_{\epsilon}(t, y), & & x \in \mathbb{R}^{n} .
\end{aligned}\right.
$$

Remark 2.2. This time the initial function affects the PDE differently (i.e. through both $f$ and $\mathcal{A} f$ ). We also comment that the last two terms in the PDE (the bi-Laplacian and the Laplacian of the solution $u_{\epsilon}$ ) look like those in a linearized Cahn-Hilliard equation with the correct $\epsilon$-scaling, but with the opposite sign for $\Delta$.

Proof. It is enough to prove the CTP case. Let

$$
u_{\epsilon}(t, x)=E\left[f\left(Z_{\epsilon C}^{x}(t)\right) \exp \left(-\frac{|C(t)|}{\epsilon}\right)\right]
$$

and

$$
v_{\epsilon}(s, x)=E\left[f\left(X^{x}(\epsilon s)\right) \exp \left(-\frac{s}{\epsilon}\right)\right]=\exp \left(-\frac{s}{\epsilon}\right) \mathcal{T}_{\epsilon s} f(x) .
$$

We then have

$$
u_{\epsilon}(t, x)=2 \int_{0}^{\infty} v_{\epsilon}(s, x) p_{t}^{1}(0, s) d s .
$$

The rest of the proof is similar to the proof of Thereom 1.2 in Allouba [2].

The next result gives a Feynman-Kac type formula for CTP's and connects it to fourth order PDE's.

Theorem 2.3. Assume that $f, c: \mathbb{R}^{n} \rightarrow \mathbb{R}$ are bounded, $c \leq 0$, and $D_{i j} f$ and $D_{i j} c$ are bounded and Hölder continuous with exponent $0<\beta \leq 1$, for $1 \leq i, j \leq n$. If the $\left|D_{i, j} v(s, x)\right| \leq K_{T}$, for all $(s, x) \in[0, T] \times \mathbb{R}^{n}$, for any time $T>0$, for all $i, j$, where $K_{T}>0$ is a constant depending only on $T$ and

$$
v(s, x)=E\left[f\left(X^{x}(s)\right) \exp \left(\int_{0}^{s} c\left(X^{x}(r)\right) d r\right)\right]
$$

where $X^{x}$ is an $n$-dimensional Brownian motion starting at $x$, then

$$
u(t, x)=E\left[f\left(Z_{C}^{x}(t)\right) \exp \left(\int_{0}^{|C(t)|} c\left(X^{x}(r)\right) d r\right)\right]
$$


solves

$$
\left\{\begin{aligned}
\frac{\partial^{2}}{\partial t^{2}} u(t, x)= & -\frac{2}{\pi t}[\Delta f(x)+c(x) f(x)] \\
& -\left[\Delta c(x)+c^{2}(x)\right] u(t, x)-2 \nabla c(x) . \nabla u(t, x) \\
& -c(x) \Delta u(t, x)-\Delta^{2} u(t, x), \quad t>0, x \in \mathbb{R}^{n}, \\
u(0, x)= & f(x)=\lim _{t \downarrow 0, y \rightarrow x} u(t, y), \quad x \in \mathbb{R}^{n} .
\end{aligned}\right.
$$

Remark 2.3. Again the effect of the initial function is through $\Delta$ and $\Delta^{2}$. The effect of the initial function fades away as $t \rightarrow \infty$ at a rate of $2 / \pi t$.

Proof. Let $u$ and $v$ be defined as in (2.10) and (2.9), respectively. Then

$$
u(t, x)=2 \int_{0}^{\infty} p_{t}^{1}(0, s) v(s, x) d s .
$$

Since $X^{x}$ is a Brownian motion starting at $x$, we have

$$
\frac{\partial}{\partial s} v(s, x)=\Delta v(s, x)+c(x) v(s, x), \text { in }(0, \infty) \times \mathbb{R}^{n} .
$$

The rest of the proof is similar to the proof of Thereom 1.3 in Allouba [2].

Now we define an iterated process motivated by the article of Allouba [3] that leads to the study of Linearized Kuramoto-Sivashinsky PDE. We call the process an imaginary-Cauchy-time-Brownian-angle process (ICTBAP). The definition needs the introduction of complex imaginary number $i=\sqrt{-1}$.

Let $f: \mathbb{R}^{n} \rightarrow \mathbb{R}$

$$
\mathcal{B}_{C}^{f, X}(t, x)= \begin{cases}f\left(X^{x}(i C(t))\right) \exp (i C(t)), & C(t) \geq 0 \\ f\left(X^{-i x}(-i C(t))\right) \exp (i C(t)), & C(t)<0\end{cases}
$$

where $X^{x}$ is an $\mathbb{R}^{n}$-valued Brownian motion starting from $x \in \mathbb{R}^{n}, X^{-i x}$ is an independent $i \mathbb{R}^{n}$-valued BM starting at $-i x$, and both are independent of the inner $\mathbb{R}$-valued Cauchy process $C$. We think of the imaginary-time processes $\left\{X^{x}(i s), s \geq\right.$ $0\}$ and $\left\{i X^{-i x}(-i s), s \leq 0\right\}$ as having the same complex Gaussian distribution on $\mathbb{R}^{n}$ with the corresponding complex distributional density

$$
p_{i s}^{n}(x, y)=\frac{1}{(4 \pi i s)^{n / 2}} e^{-|x-y|^{2} / 4 i s} .
$$

The space derivative terms in the PDE in the following theorem is the same as in a linearized Kuramoto-Sivashinsky PDE.

Theorem 2.4. Let $f \in C_{c}^{2}\left(\mathbb{R}^{n} ; \mathbb{R}\right)$ with $D_{i j} f$ Hölder continuous with exponent $0<\beta \leq 1$, for all $1 \leq i, j \leq n$. Let

$$
\begin{gathered}
v(s, x)=\exp (i s) \int_{\mathbb{R}^{n}} f(y) \frac{1}{(4 \pi i s)^{n / 2}} e^{-|x-y| / 4 i s} d y, \\
u(t, x)=\int_{-\infty}^{0} v(s, x) p_{t}^{1}(0, s) d s+\int_{0}^{\infty} v(s, x) p_{t}^{1}(0, s) d s,
\end{gathered}
$$

where

$$
p_{t}^{1}(0, s)=\frac{t}{\pi\left(s^{2}+t^{2}\right)}
$$


is the transition density of the inner (one-dimensional) Cauchy process. Then $u(t, x)$ solves

$$
\begin{cases}\frac{\partial^{2}}{\partial t^{2}} u(t, x)=\Delta^{2} u(t, x)+2 \Delta u(t, x)+u(t, x), & t>0, x \in \mathbb{R}^{n}, \\ u(0, x)=f(x), & x \in \mathbb{R}^{n} .\end{cases}
$$

Proof. We comment that

$$
v(s, x)=E\left[f\left(X^{x}(i s)\right) \exp (i s)\right]
$$

and

$$
u(t, x)=E\left[\mathcal{B}_{C}^{f, X}(t, x)\right]
$$

The proof is the same as in [3] except that the start point $p_{t}^{1}(0, s)$ satisfies

$$
\left(\frac{\partial^{2}}{\partial s^{2}}+\frac{\partial^{2}}{\partial t^{2}}\right) p_{t}^{1}(0, s)=0
$$

which explains the sign change from Theorem 1.1 in [3]. We can differentiate under the integral by Lemma 2.1 in [3] and Lemma 3.1. The rest of the proof is similar to the proof of Thereom 1.1 in Allouba [3].

For $\alpha \neq 1$, the PDE is more complicated since kernels of symmetric $\alpha$-stable processes satisfy a higher order PDE. We also have to assume that we can integrate under the integral as much as we need in the case where the outer process is BM (or in general we can take the operator out of the integral). This is valid for $\alpha=1 / m$, $m=2,3, \cdots$, by Lemma 3.3 below.

Theorem 2.5. Let $\alpha \in(0,2)$ be rational $\alpha=l / m$, where $l$ and $m$ are relatively prime. Let $\mathcal{T}_{s} f(x)=E\left[f\left(X^{x}(s)\right)\right]$ be the semigroup of the continuous Markov process $X^{x}(t)$ and let $\mathcal{A}$ be its generator. Let $f$ be a bounded measurable function in the domain of $\mathcal{A}$, with $D^{\gamma} f$ bounded and Hölder continuous for all multi-index $\gamma$ such that $|\gamma|=2 l$. If $u(t, x)=E\left[f\left(Z_{Y, c}^{x, k}(t)\right)\right]$ for any $k \in \mathbb{N}$, then $u$ solves the PDE

$$
\begin{aligned}
(-1)^{l+1} \frac{\partial^{2 m}}{\partial t^{2 m}} u(t, x)= & -2 \sum_{i=1}^{l}\left(\left.\frac{\partial^{2 l-2 i}}{\partial s^{2 l-2 i}} p_{t}^{\alpha}(0, s)\right|_{s=0}\right) \mathcal{A}^{2 i-1} f(x) \\
& -\mathcal{A}^{2 l} u(t, x), \quad t>0, \quad x \in \mathbb{R}^{n}, \\
u(0, x)= & f(x), \quad x \in \mathbb{R}^{n},
\end{aligned}
$$

where the operator $\mathcal{A}$ acts on $u(t, x)$ as a function of $x$ with $t$ fixed. In particular, if $X^{x}(t)$ is Brownian motion started at $x$ and $\Delta$ is the standard Laplacian, then $u$ solves

$$
\begin{aligned}
(-1)^{l+1} \frac{\partial^{2 m}}{\partial t^{2 m}} u(t, x)= & -2 \sum_{i=1}^{l}\left(\left.\frac{\partial^{2 l-2 i}}{\partial s^{2 l-2 i}} p_{t}^{\alpha}(0, s)\right|_{s=0}\right) \Delta^{2 i-1} f(x) \\
& -\Delta^{2 l} u(t, x), \quad t>0, \quad x \in \mathbb{R}^{n}, \\
u(0, x)= & f(x), \quad x \in \mathbb{R}^{n} .
\end{aligned}
$$

Proof. We use the representation

$$
u(t, x)=E\left[f\left(Z_{Y}^{x}(t)\right)\right]=2 \int_{0}^{\infty} p_{t}^{\alpha}(0, s) \mathcal{T}_{s} f(x) d s
$$


and the fact that the transition density of the process $Y(t)$ satisfies

$$
\left[\left(\frac{\partial^{2}}{\partial s^{2}}\right)^{l}+(-1)^{l+1}\left(\frac{\partial^{2 m}}{\partial t^{2 m}}\right)\right] p_{t}^{\alpha}(0, s)=0, \quad(t, x) \in(0, \infty) \times \mathbb{R}^{n},
$$

from Lemma 3.2. Then, we use integration by parts, as many as needed, to pass the $s$ derivatives to $\mathcal{T}_{s} f(x)$ from $p_{t}^{\alpha}(0, s)$. The boundary terms are all zero at $\infty$ (as $s$ goes to $\infty$ ). Also, all the odd $s$ derivatives of $p_{t}^{\alpha}(0, s)$ at 0 are zero.

\section{USEFUl LEMMAS}

We start with a result that is useful in taking the derivative under the integral. With the following lemma we can now establish similar results to the ones in [1] and $[2]$.

Lemma 3.1. Let $X^{x}$ be an $n$-dimensional Brownian motion starting at $x$, and let $f, g: \mathbb{R}^{n} \rightarrow \mathbb{R}$ be bounded and measurable such that $D_{i j} f$ and $D_{i j} g$ are Hölder continuous, with exponent $0<\beta \leq 1$, for $1 \leq i, j \leq n$. Let

$$
\begin{gathered}
u_{1}(t, x)=\int_{0}^{\infty} E\left[f\left(X^{x}(s)\right)\right] p_{t}^{1}(0, s) d s, \\
u_{2}(t, x)=\int_{0}^{t} \int_{0}^{\infty} E\left[g\left(X^{x}(s)\right)\right] p_{t}^{1}(0, s) d s d r .
\end{gathered}
$$

Then $\Delta^{2} u_{1}(t, x)$ and $\Delta^{2} u_{2}(t, x)$ are finite and

$$
\begin{gathered}
\Delta^{2} u_{1}(t, x)=\int_{0}^{\infty} \Delta^{2} E\left[f\left(X^{x}(s)\right)\right] p_{t}^{1}(0, s) d s \\
\Delta^{2} u_{2}(t, x)=\int_{0}^{t} \int_{0}^{\infty} \Delta^{2} E\left[g\left(X^{x}(s)\right)\right] p_{t}^{1}(0, s) d s d r .
\end{gathered}
$$

Proof. The proof follows from the proof of Lemma 2.1 in [2] with only changing the density of time process

$$
p_{t}^{1}(0, s)=\frac{t}{\pi\left(s^{2}+t^{2}\right)},
$$

which is the transition density of the real-valued Cauchy process. Our results follow from the facts

$$
\int_{0}^{\infty} p_{t}^{1}(0, s) s^{1-\beta / 2} d s=\int_{0}^{\infty} \frac{t}{\pi\left(s^{2}+t^{2}\right) s^{1-\beta / 2}} d s<\infty
$$

and

$$
\int_{0}^{t} \int_{0}^{\infty} p_{r}^{1}(0, s) / s^{1-\beta / 2} d s d r=\int_{0}^{t} \int_{0}^{\infty} \frac{r}{\pi\left(s^{2}+r^{2}\right) s^{1-\beta / 2}} d s d r<\infty
$$

For $\alpha \in(0,2)$ and $\alpha \neq 1$, we have the following lemma from DeBlassie [8, Theorem 1.1].

Lemma 3.2. Let $\alpha=l / m$ with $l, m$ relatively prime. The transition density $p_{t}^{\alpha}(x, y)$ of the $\alpha$-stable process satisfies the PDE for $y$ fixed

$$
\left(\Delta^{l}+(-1)^{l+1} \frac{\partial^{2 m}}{\partial t^{2 m}}\right) p_{t}^{\alpha}(x, y)=0, \quad(t, x) \in(0, \infty) \times \mathbb{R}^{n} .
$$

The next lemma allows us to take the bi-Laplacian out of the integral in Theorem 2.5 for some values of the index of the symmetric stable process. 
Lemma 3.3. Let $X^{x}$ be an $n$-dimensional Brownian motion starting at $x$, and let $f: \mathbb{R}^{n} \rightarrow \mathbb{R}$ be bounded and measurable such that $D_{i j} f$ is Hölder continuous, with exponent $0<\beta \leq 1$, for $1 \leq i, j \leq n$. Let $\alpha=1 / m, m=2,3, \cdots$, and

$$
u(t, x)=\int_{0}^{\infty} E\left[f\left(X^{x}(s)\right)\right] p_{t}^{\alpha}(0, s) d s .
$$

Then $\Delta^{2} u(t, x)$ is finite and

$$
\Delta^{2} u(t, x)=\int_{0}^{\infty} \Delta^{2} E\left[f\left(X^{x}(s)\right)\right] p_{t}^{\alpha}(0, s) d s .
$$

Proof. The proof follows similarly to the proof of Lemma 3.1, by showing that

$$
\int_{0}^{\infty} p_{t}^{\alpha}(0, s) s^{1-\beta / 2} d s=\int_{0}^{\infty} \int_{0}^{\infty} \frac{p_{l}(0, s) u_{t}^{\alpha / 2}(l)}{s^{1-\beta / 2}} d l d s<\infty .
$$

Above we use the fact that

$$
p_{t}^{\alpha}(0, s)=\int_{0}^{\infty} p_{l}(0, s) u_{t}^{\alpha / 2}(l) d l,
$$

where $p_{l}(0, s)$ is the density of real-valued Brownian motion, and $u_{t}^{\alpha / 2}(l)$ is the density of the $\alpha / 2$ subordinator (see [8] for this representation and the bounds for integrals of $\left.u_{t}^{\alpha / 2}(l)\right)$.

\section{Subordinate killed Brownian motion}

Let $D \subset \mathbb{R}^{n}$ be a bounded domain and let $X_{t}^{D}$ be the Brownian motion killed upon exiting $D$. The subordinate killed Brownian motion $Z_{t}^{D}$ is defined as the process obtained by subordinating $X^{D}$ via the $\alpha / 2$-subordinator $T_{t}$. More precisely, let $Z_{\alpha}^{D}=X^{D}\left(T_{t}\right), t>0$. Then $Z_{\alpha}^{D}$ is a Hunt process on $D$. If we use $\left(P_{t}^{D}\right)_{t \geq 0}$ to denote the semigroup of $X^{D}$, then the semigroup $Q_{t}^{\alpha}$ of $Z_{\alpha}^{D}$ is given by

$$
Q_{t}^{\alpha} f(x)=\int_{0}^{\infty} P_{s}^{D} f(x) u_{t}^{\alpha / 2}(s) d s .
$$

The following theorem establishes the PDE connection of subordinate killed Brownian motion.

Theorem 4.1. Let $0<\alpha=\frac{k}{m}<2$ where $k, m$ are relatively prime integers. Let $f: D \rightarrow \mathbb{R}$ be a bounded function. Then $u(t, x)=Q_{t}^{\alpha} f(x)$ solves the following $P D E$ :

$$
\begin{cases}\Delta^{k} u(t, x)+(-1)^{k+1} \frac{\partial^{2 m}}{\partial t^{2 m}} u(t, x)=0, & (t, x) \in(0, \infty) \times D, \\ u(0, x)=f(x), & x \in D, \\ u(t, x)=0, & x \in \partial D .\end{cases}
$$

To prove Theorem 4.1, we need a passing derivative under the integral lemmas.

Lemma 4.1. Let $u(t, x)$ be as in Theorem 4.1 ; then

$$
\Delta^{k} u(t, x)=\int_{0}^{\infty} \Delta_{x}^{k} P_{s}^{D} f(x) u_{t}^{\alpha / 2}(s) d s .
$$


Proof. We know that

$$
\Delta^{k} P_{s}^{D} f(x)=\sum_{l=1}^{\infty}\left(-\lambda_{l}\right)^{k} e^{-s \lambda_{l}} \varphi_{l}(x) \int_{D} \varphi_{l}(y) d y .
$$

Now

$$
\begin{aligned}
\Delta^{k} u(t, x) & =\int_{0}^{\infty} \Delta^{k} P_{s}^{D} f(x) u_{t}^{\alpha / 2}(s) d s \\
& =\sum_{l=1}^{\infty}\left(-\lambda_{l}\right)^{k} \varphi_{l}(x) \int_{D} \varphi_{l}(y) d y \int_{0}^{\infty} e^{-s \lambda_{l}} u_{t}^{\alpha / 2}(s) d s \\
& =\sum_{l=1}^{\infty}\left(-\lambda_{l}\right)^{k} e^{-t\left(\lambda_{l}\right)^{\alpha / 2}} \varphi_{l}(x) \int_{D} \varphi_{l}(y) d y
\end{aligned}
$$

where (4.1) follows because the Laplace transform of the density of $T_{t}$ is

$$
\int_{0}^{\infty} e^{-s \lambda} u_{t}^{\alpha / 2}(s) d s=e^{-t \lambda^{\alpha / 2}}, \text { for } \lambda>0 .
$$

This last series is absolutely and uniformly convergent. Indeed let $\delta>0$; since $p_{s}^{D}(x, y) \leq(4 \pi s)^{-n / 2}$, for all $s>0$ we see that

$$
\begin{aligned}
\left|e^{-s \lambda_{l}} \varphi_{l}(x)\right| & =\left|\int_{D} p_{s}^{D}(x, y) \varphi_{l}(y) d y\right| \\
& \leq(4 \pi s)^{-n / 2}\left\|\varphi_{l}\right\|_{2}(\operatorname{vol}(D))^{1 / 2} \\
& =(4 \pi s)^{-n / 2}(\operatorname{vol}(D))^{1 / 2} .
\end{aligned}
$$

Taking $s=1 / \lambda_{l}$ gives that

$$
\left|\varphi_{l}(x)\right| \leq e(4 \pi)^{-n / 2} \lambda_{l}^{n / 2}[\operatorname{vol}(D)]^{1 / 2},
$$

for all $x \in D$. Now since the volume of the domain $D$ is finite, there exists $C>0$ such that

$$
\sum_{l=1}^{\infty}\left(-\lambda_{l}\right)^{k} e^{-t\left(\lambda_{l}\right)^{\alpha / 2}} \varphi_{l}(x) \int_{D} \varphi_{l}(y) d y \leq C \sum_{l=1}^{\infty} e^{-\delta \lambda_{l}^{\alpha / 2} / 2},
$$

for all $(t, x) \in[0, \infty) \times D$ and since the last series is convergent by Weyl's asymptotic lemma (see [7]) we have $c_{n, D} l^{n / 2} \leq \lambda_{l}$ where $c_{n, D}$ is a constant that depends on $n$ and the volume of the domain $D$.

Lemma 4.2. Suppose $f$ is bounded. Set $u(t, x)=Q_{t}^{\alpha} f(x)$. Then for any integer $q \geq 0$,

$$
\frac{\partial^{q}}{\partial t^{q}} u(t, x)=\int_{D} \int_{0}^{\infty} f(y) p_{s}^{D}(x, y) \frac{\partial^{q}}{\partial t^{q}} u_{t}^{\alpha / 2}(s) d s d y .
$$

Proof. The proof is the same as in Lemma 2.1 in [8] almost word for word except we use the fact that $\int_{D} p_{s}^{D}(x, y) d y=1$.

The next lemma is Lemma 3.1 in [8]; we give it for completeness.

Lemma 4.3. Let $\alpha=k / m$. For $s$ and $t$,

$$
\left(\frac{\partial^{k}}{\partial s^{k}}-\frac{\partial^{2 m}}{\partial t^{2 m}}\right) u_{t}^{\alpha / 2}(s)=0 .
$$


Proof of Theorem 4.1. We first apply Lemma 4.1, then integration by parts repeatedly, and appealing equations (2.7)-(2.10) in [8], we see the boundary terms are all 0 :

$$
\begin{aligned}
\Delta^{k} u(t, x) & =\int_{D} \int_{0}^{\infty} f(y) \Delta_{x}^{k} p_{s}^{D}(x, y) u_{t}^{\alpha / 2}(s) d s d y \\
& =\int_{D} \int_{0}^{\infty} f(y)\left[\frac{\partial^{k}}{\partial s^{k}} p_{s}^{D}(x, y)\right] u_{t}^{\alpha / 2}(s) d s d y \\
& =\int_{D}-f(y) \int_{0}^{\infty}\left[\frac{\partial^{k-1}}{\partial s^{k-1}} p_{s}^{D}(x, y)\right] \frac{\partial}{\partial s} u_{t}^{\alpha / 2}(s) d s d y \\
& =\cdots=(-1)^{k} \int_{D} \int_{0}^{\infty} f(y) p_{s}^{D}(x, y) \frac{\partial^{k}}{\partial s^{k}} u_{t}^{\alpha / 2}(s) d s d y .
\end{aligned}
$$

By Lemma 4.2,

$$
\frac{\partial^{q}}{\partial t^{q}} u(t, x)=\int_{D} \int_{0}^{\infty} f(y) p_{s}^{D}(x, y) \frac{\partial^{q}}{\partial t^{q}} u_{t}^{\alpha / 2}(s) d s d y
$$

So we get

$$
\begin{aligned}
\Delta^{k} u(t, x)+(-1)^{k+1} \frac{\partial^{2 m}}{\partial t^{2 m}} u(t, x) & =\int_{D} \int_{0}^{\infty} f(y)\left[(-1)^{k} \frac{\partial}{\partial s^{k}} u_{t}^{\alpha / 2}(s)\right. \\
& \left.+(-1)^{k+1} \frac{\partial^{2 m}}{\partial t^{2 m}} u_{t}^{\alpha / 2}(s)\right] p_{s}^{D}(x, y) d s d y \\
& =0, \text { by Lemma 4.3. }
\end{aligned}
$$

Now using dominated convergence theorem, we see that the boundary conditions are satisfied.

\section{ACKNOWLEDGMENTS}

The author would like to thank Professor Rodrigo Bañuelos, his academic advisor, for his guidance on this paper.

\section{References}

[1] H. Allouba, W. Zheng, Brownian-time processes: The PDE connection and the halfderivative generator, Ann. Prob. 29 (2001), 1780-1795. MR1880242 (2002j:60118)

[2] H. Allouba, Brownian-time process: The PDE connection II and the corresponding FeynmanKac formula, Trans. Amer. Math. Soc. 354 (2002), 4627-4637. MR1926892 (2003m:60177)

[3] H. Allouba, A linearized Kuramoto-Sivashinsky PDE via an imaginary-Brownian-timeBrownian-angle process, C.R. Acad. Sci. Paris Ser. 1336 (2003), 309-314. MR1976309 (2004b:60197)

[4] K. Burdzy, Some path properties of iterated Brownian motion, In Seminar on Stochastic Processes (E. Çinlar, K.L. Chung and M.J. Sharpe, eds.), Birkhäuser, Boston (1993), 67-87. MR1278077 (95c:60075)

[5] K. Burdzy, Variation of iterated Brownian motion, In Workshops and Conference on Measurevalued Processes, Stochastic Partial Differential Equations and Interacting Particle Systems (D.A. Dawson, ed.) Amer. Math. Soc. Providence, RI (1994), 35-53. MR1278281 (95h:60123)

[6] K. Burdzy and D. Khoshnevisan, Brownian motion in a Brownian crack, Ann. Appl. Probabl. 8 (1998), no. 3, 708-748. MR1627764 (99g:60147)

[7] I. Chavel, Eigenvalues in Riemannian geometry, Academic Press, 1984. MR768584 (86g:58140)

[8] R. D. DeBlassie, Higher order PDE's and Symmetric Stable Processes, Probab. Theory Relat. Fields 129 (2004), 495-536. MR2078980 (2005d:60079) 
[9] R. D. DeBlassie, Iterated Brownian motion in an open set, Ann. Appl. Prob. 14 (2004), no. 3, 1529-1558. MR2071433 (2005f:60172)

[10] T. Funaki, A probabilistic construction of the solution of some higher order parabolic differential equations, Proc. Japan Acad. Ser. A. Math. Sci. 55 (1979), 176-179. MR533542 (80h:60075)

[11] R. Getoor, First passage times for symmetric stable processes in space, Trans. Amer. Math. Soc. 101 (1961), 75-90. MR0137148 (25:604)

[12] D. Khoshnevisan and T.M. Lewis, Chung's law of the iterated logarithm for iterated Brownian motion, Ann. Inst. H. Poincaré Probab. Statist. 32 (1996), no. 3, 349-359. MR1387394 (97k:60218)

[13] E. Nane, Iterated Brownian motion in parabola-shaped domains, Potential Analysis, 24 (2006), 105-123. MR2217416

[14] R. Song, Sharp bounds on the density, Green function and jumping function of subordinate killed BM, Probab. Theory Relat. Fields 128 (2004), 606-628. MR2045955 (2005c:60099)

[15] R. Song and Z. Vodraček, Potential Theory of subordinate killed Brownian motion in a domain, Probab. Theory Relat. Fields 125 (2003), 578-592. MR1974415 (2004g:60110)

[16] Y. Xiao, Local times and related properties of multidimensional iterated Brownian motion, J. Theoret. Probab. 11 (1998), no. 2, 383-408. MR1622577 (99g:60136)

Department of Mathematics, Purdue University, West Lafayette, Indiana 47906

E-mail address: enane@math.purdue.edu

Current address: Department of Statistics and Probability, A 413 Wells Hall, Michigan State University, East Lansing, Michigan 48824 